\title{
Structural Analysis of Exosomes Using Different Types of Electron Microscopy
}

\author{
Hyosun Choi ${ }^{1}$, Ji Young Mun ${ }^{1,2, *}$ \\ ${ }^{1}$ BK21 Plus Program, Department of Senior Healthcare, Graduate School, Eulji University, Daejeon 34824, Korea \\ ${ }^{2}$ Department of Biomedical Laboratory Science, College of Health Science, Eulji University, Seongnam 13135, Korea
}

*Correspondence to:

Mun JY,

Tel: +82-31-740-7380

Fax: +82-31-740-7354

E-mail: mjy1026@gmail.com

Received June 12, 2017

Revised July 24, 2017

Accepted July 24, 2017
Negative staining has been traditionally used for exosome imaging; however, the technique is limited to surface topology only and can cause staining artifacts. Therefore, to analyze the internal structure of exosomes, we employed a method of block preparation, thin sectioning, and electron tomography. In addition, an automatic serial sectioning technique with 15-nm thickness through focused ion beam was employed to observe the three-dimensional structure of exosomes of various sizes. Cryo-transmission electron microscopy revealed the near-to-native structure of exosomes.

Key Words: Exosome, Electron tomography, Serial block face section through focused ion beam, Cryo-transmission electron microscopy

\section{INTRODUCTION}

Interest in exosomes as biomarkers for early diagnosis of a disease is rapidly increasing because exosomes contain specific proteins or genetic material that represent the functions of their original cells (Thery, 2011). It is not easy to characterize exosomes because among extracellular vesicles, shed microvesicles $(100 \sim 1,000 \mathrm{~nm})$ have a size similar to exosomes (40 150 nm) (Villarroya-Beltri et al., 2014; Vlassov et al., 2012). In addition, because exosomes have various sizes and morphologies depending on their origin and function, they need to be analyzed using electron microscopy (EM). Currently, the cup shape of exosomes identified through negative staining has been considered to be their typical structure (Gyorgy et al., 2011; Thery et al., 2009). However, scanning EM (SEM) (Enderle et al., 2015), atomic force microscopy (Sharma et al., 2010), and cryo-transmission EM (TEM) (Montaner-Tarbes et al., 2016; Saari et al., 2015) revealed that exosomes have a round shape. In this study, we investigated the structure of exosomes using various EM approaches, including block preparation, sectioning, focused ion beam (FIB)-SEM, electron tomography, and cryo-TEM.

\section{MATERIALS AND METHODS}

\section{Block Preparation for Sectioning of Exosomes}

Pellets of exosomes were fixed with $2.5 \%$ glutaraldehyde in $0.1 \mathrm{M}$ cacodylate solution $(\mathrm{pH} 7.0)$ for 1 hour and then in $2 \%$ osmium tetroxide for 1 hour at $4^{\circ} \mathrm{C}$. They were then dehydrated using a graded acetone series and embedded in Spurr's medium (Electron Microscopy Sciences). Samples were sectioned $(60 \mathrm{~nm})$ using an ultramicrotome (RMC MTXL, USA) and were double stained with $2 \%$ uranyl acetate for 20 minutes and lead citrate for 10 minutes. The sections were then viewed under the Hitachi H-7600 TEM (Hitachi, Japan) at $80 \mathrm{kV}$.

\section{Serial Sections for Three-Dimensional Reconstruction}

The exosome blocks were mounted on an aluminum stub with conductive silver paint and were then coated with Pt using a sputter coater to remove the charge. In the regions selected by surface block face (SBF), a protective layer of carbon was deposited to reduce milling artifacts and were polished using a less powerful ion beam current. Continuous milling was performed to obtain 15-nm-thick sections, which were imaged using the FIB-SEM microscope $\left(\mathrm{AUGIRA}^{\mathrm{TM}}\right.$; Carl

(a) This is an open-access article distributed under the terms of the Creative Commons Attribution Non-Commercial License (http://creativecommons.org/licenses/by-nc/4.0) which permits unrestricted noncommercial use, distribution, and reproduction in any medium, provided the original work is properly cited.

Copyrights @ 2017 by Korean Society of Microscopy 
Zeiss NTS GmbH, Germany that was installed at Gyeongbuk Science \& Technology Promotion Center in Gumi) and a backscattered electron detector with an In-lens EsB detector for high-resolution and contrast imaging (GEMINI ${ }^{\circledR}$ FE-SEM column; Carl Zeiss NTS GmbH). The milling and imaging were repeated continuously in a completely automated manner, with $1.8-\mathrm{kV}$ acceleration voltage for serial sections. Serial images were aligned using the ETOMO software (University of Colorado; http://bio3d.colorado.edu/imod/ doc/etomoTutorial.html), and the contours of the exosomes were obtained using the IMOD program (http://bio3d. colorado.edu/imod/) for three-dimensional (3D) structure reconstruction.

\section{Electron Tomography of Exosome-Treated Cells}

To investigate the structure of exosomes in target cells, cells were treated with exosomes for 30 minutes and were then fixed with $2.5 \%$ glutaraldehyde in $0.1 \mathrm{M}$ cacodylate solution ( $\mathrm{pH} 7.0$ ) for 1 hour, followed by $2 \%$ osmium tetroxide for 1 hour at $4^{\circ} \mathrm{C}$. They were dehydrated using a graded acetone series and embedded in Spurr's medium. Samples were sectioned $(60 \mathrm{~nm})$ using an ultramicrotome and were double stained with $2 \%$ uranyl acetate for 20 minutes and lead citrate for 10 minutes. Tilt images at intervals of $2^{\circ}$ at $\pm 60^{\circ}$ were obtained using the JEM2200FS microscope (JEOL, Japan; installed at the National Institute for Physiological Sciences in Okazaki) with a CCD camera (F415; TVPIS, Germany). Alignment and tomograms were obtained using the ETOMO software.

\section{Cryofixation with a High-Pressure Freezer: Freeze Substitution After Exosome Treatment of Cells}

Pellets were placed in a cavity filled with $20 \%$ bovine serum albumin, were rapidly loaded in a high-pressure freezer (HPF, HPM100; Leica Microsystems, Austria), and cryoimmobilized in liquid nitrogen. The samples were removed from the liquid nitrogen and were transferred to the freeze-substitution (FS) apparatus (AFS, Leica Microsystems). The tubes filled with $2 \%$ osmium tetroxide in acetone were precooled for FS. The specimens are washed thrice with anhydrous acetone and were infiltrated with Spurr's resin $(30 \%, 70 \%$, and $100 \%$ resin). Polymerization was performed with fresh resin at $60^{\circ} \mathrm{C}$. Samples were sectioned $(60 \mathrm{~nm})$ using an ultramicrotome and were double stained with $2 \%$ uranyl acetate for 20 minutes and lead citrate for 10 minutes. The sections were imaged using the Hitachi H-7600 TEM at $80 \mathrm{kV}$.

\section{Cryo-TEM of Exosomes}

For cryo-TEM of the samples, carbon grids with a hydrophilic surface were prepared with a plasma cleaning system (JEOL), and $5 \mu \mathrm{L}$ of exosomes were added onto the grid and frozen using Vitrobot (FEI, The Netherlands) in liquid nitrogen.
The samples were analyzed with the FEI Talos L120C cryoTEM (FEI) at the Nanobio Imaging Center, Seoul National University.

\section{RESULTS AND DISCUSSION}

\section{Exosomes from Block Sectioning}

Exosomes have been observed as "whole-mount" samples when deposited on EM grids without sectioning. Cup-shaped structures have been considered to be the characteristic of exosomes through negative stain TEM; however, frozen exosomes with cryo-TEM showed round-shaped structures (Raposo \& Stoorvogel, 2013). To elucidate the more natural structure of exosomes, we first checked the typical cup-shaped structure through negative staining. We then attempted block preparation with chemical fixation and HPF-FS, serial sectioning, and cryo-TEM. To reduce possible artifacts from dehydration, we prepared blocks using the conventional chemical EM fixation method or cryo-immobilization. HPFFS has been considered to be a useful fixation technique because it can reduce changes in the middle of structures during fixation and dehydration. For chemical fixation, glutaraldehyde and osmium acid were used, then graded dehydration and plastic embedding were performed at $60^{\circ} \mathrm{C}$ using epoxy resin. Ultrathin sections of blocks prepared using the conventional (Fig. 1A) or cryofixation (Fig. 1B) method showed round exosomes instead of a cup shape.

\section{Serial Sectioning and Electron Tomography}

Serial sectioning helps to understand the 3D structure of exosomes and their structure in target cells. Traditional ultramicrotomy does not require specific equipment beyond an ultramicrotome; however, an experienced person has to spend a long time preparing serial sections without loss of even single sections. In addition, imaging of serial sections is
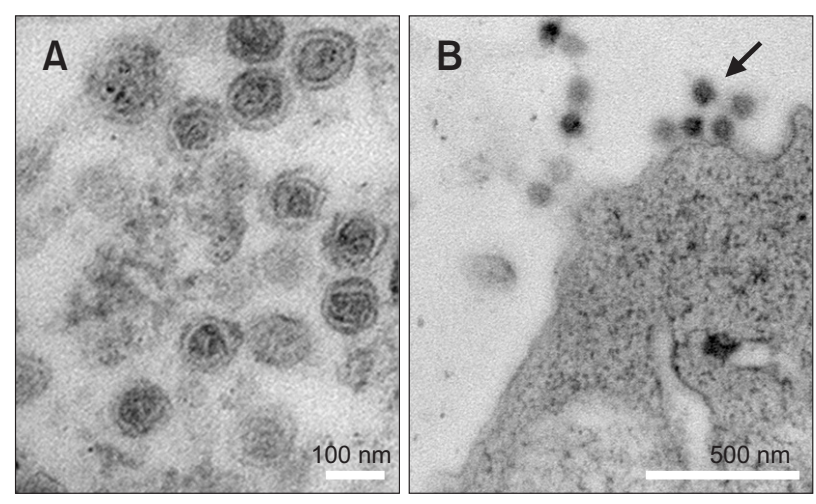

Fig. 1. Sectioned images of exosomes after block preparation. (A) Cryotransmission electron microscopy (TEM) image from a chemically fixed block. (B) TEM image from an HPF-FS block of exosome-treated cells. Arrow indicates exosome. 
time-consuming work, and every image should be carefully aligned and stacked using a specific software. With recent progress in EM techniques, we are now able to obtain serial images automatically through several methods (Choi et al., 2017; Titze \& Genoud, 2016). In SBF-SEM, because cutting and imaging of a plastic-embedded block surface, directly mounted inside SEM, are integrated in the equipment, alignment and contamination issues can be eliminated. The surface of the block is cut using a microtome in the $3 \mathrm{VEW}$ system or Volume Scope and using FIB in FIB-SEM (Titze \& Genoud, 2016). Imaging and cutting can be repeated thousands of times. Our serial imaging at 15-nm thickness using FIB-SEM showed detailed exosome structure (Fig. 2), as well as the structure of exosomes in a target cell (data not shown). A big advantage of SBF-SEM is the good quality of serial images obtained without folding, distortion, or loss of sections. In particular, FIB was a good SBF-SEM technique for obtaining thinner sections than those obtained with other SBF techniques using a diatome blade. The 3D structure of exosomes was reported in yeast by single particle analysis of 5,000 negatively stained conical tilt pairs (Liu \& Wang, 2011). In this study, our result was the first $3 D$ structure of exosomes through serial sectioning. The contours obtained from serial sections showed that the structures of the exosome were round instead of cup shaped (Fig. 2); however, there is a resolution limitation using this method. For more detailed structure analysis, electron tomography using tilted images showed improved resolution (Fig. 3). We obtained 61 tilted

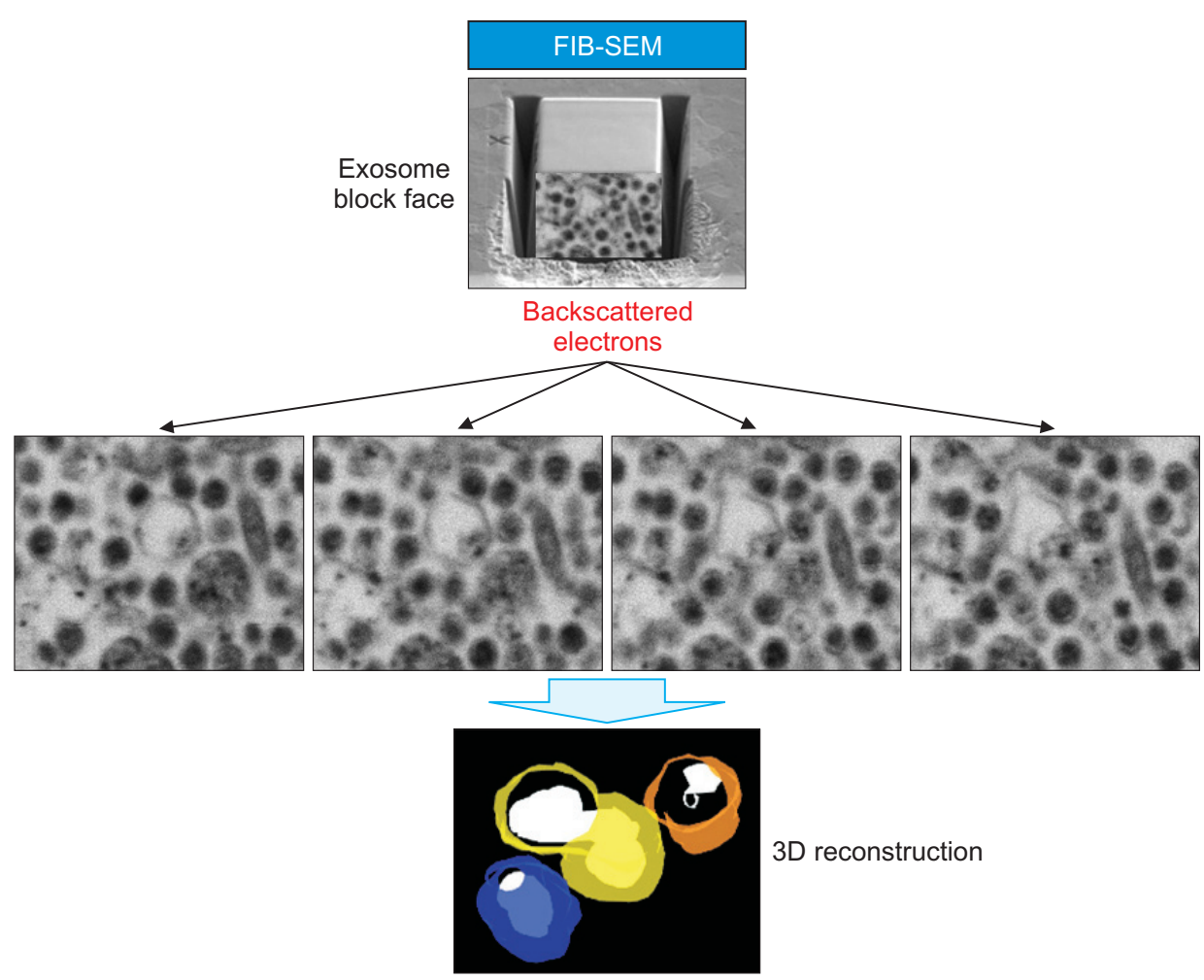

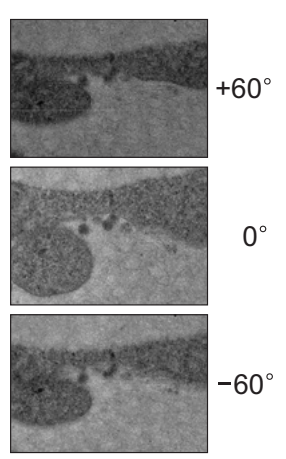

Tilted images

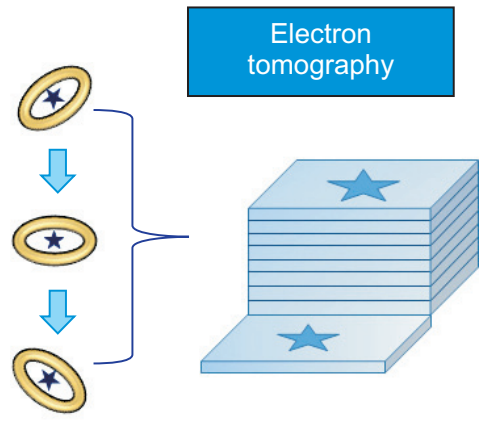

Aligned images stack

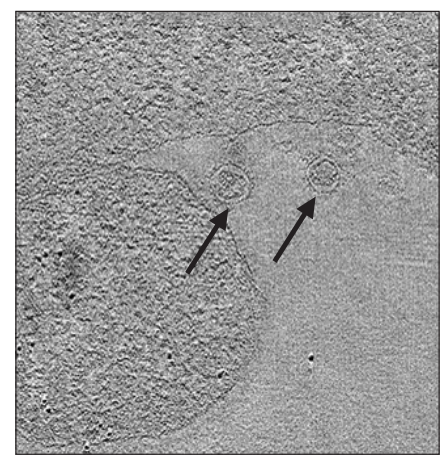

Improved resolution
Fig. 2. Three-dimensional (3D) reconstruction of exosome serial sections by focused ion beam (serial block face)SEM (FIB-SEM). The FIB-SEM setup is a combination of ion milling (by gallium ions) and SEM imaging (by backscattered electrons) in the same instrument. The sections are cut automatically using the gallium ion beam and then images of the surface block face are prepared using the backscattered electron beam. Serial sections are easy to align for 3D reconstruction because of automatic sectioning and imaging without loss of samples. The 3D structures are reconstructed using the IMOD software. Each exosome can be represented as a different color.

Fig. 3. Electron tomography. The 61 tilt images $\left(2^{\circ}\right.$ intervals at $\left.\pm 60^{\circ}\right)$ of sectioned exosomes with $60-\mathrm{nm}$ thickness were used for electron tomography. The tomogram was obtained using the through ETOMO software and showed improved resolution. Tilted images (left panel) do not show a clear detailed inner structure of exosomes, unlike the electron tomogram. Arrows show exosomes. 
images at $2^{\circ}$ intervals at $\pm 60^{\circ}$ in JEM2200FS, which were aligned and made into a tomogram using the ETOMO software. The improved resolution more clearly showed the inner structure of exosomes (Fig. 3). Serial images from FIBSEM and tilt images for tomograms showed round-shaped exosomes and their inner structure instead of cup-shaped exosomes and their surface structure.

\section{Cryofixation of Exosome-Treated Cells and Cryo-TEM of Exosomes}

Studying the biological functions of exosomes is necessary for tracing specific proteins inside exosomes. Various fluorescent dyes for exosome through labeling of specific proteins have been used, but the techniques cannot distinguish exosomes depending on their size and shape because of exaggerated fluorescence signals. Immunogold EM is mainly enables visualization of the entry and egress of exosomes in recipient cells, as well as exosomes tagged for specific proteins. Exosomes released from cancer cells show different biological functions, such as increased proliferation and invasion of other cancer cells, stimulation of endothelial cells to form tubules that support tumor angiogenesis, and inhibition of cytotoxic T lymphocytes, depending on the recipient cells (Zaborowski et al., 2015). Therefore, visualization of exosomes with specific antibody binding in cells through immunogold EM can clearly show their functions. To study the function of exosomes in cells, exosome-treated cell pellets or cell monolayers grown on coverslips (chemical fixation), aclar film (chemical and cryofixation) (Jimenez et al., 2006), or sapphire discs (particularly for cryofixation owing to good conductivity) can be used. Conventional chemical EM fixation can be used for immunostaining. However, because aldehyde and osmium tetroxide in chemical fixation usually hinders antibody binding to antigens, cryo-immobilization is better. Cryo-techniques for cells can be divided into plunge (possible depth of vitrification; $20 \mu \mathrm{m})$, jet freezing $(50 \mu \mathrm{m})$, and high-pressure freezing $(200 \mu \mathrm{m})$. Cryo-fixed cells can be sectioned by a cryo-ultramicrotome for cryo-TEM, but it is not easy to get good sections. For better sections, plastic embedding can be performed at $-35^{\circ} \mathrm{C}$ (Lowicryl embedding media), room temperature with ultraviolet light (LR White), or at $60^{\circ} \mathrm{C}$ (epoxy embedding media) with FS. In this study, FS and epoxy embedding were attempted (Fig. 1B). Plasticembedded samples for immunostaining were sectioned using an ultramicrotome (60 80-nm thickness). Sections collected using an EM grid were stained for specific antigens using antibody-conjugated gold nanoparticles (immunogold labeling) (Kim et al., 2017). In HPF-FS images, we confirmed that the shape of exosomes was round. Cryo-TEM is considered the best method for nanoparticles or proteins without dehydration artifacts. Cryo-TEM images of exosomes showed that they were of a relatively large size because they
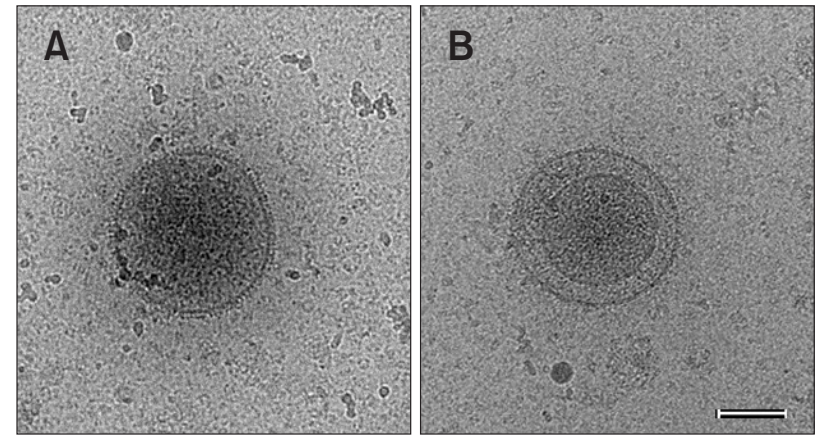

Fig. 4. Cryo-fixed exosomes. (A, B) Cryo-transmission electron microscopy images. The bar represents $100 \mathrm{~nm}$.

were hydrated structures, and structures in membranes and the lumen were clearly observed (Fig. 4).

\section{CONCLUSIONS}

Negative staining of exosomes is a conventional technique, which shows the cup shape regarded as their typical shape. However, in this study, we observed sectioned exosomes from blocks prepared using the chemical fixation method or cryofixation, which reduced dehydration defects, and hydrated exosomes by cryo-TEM. They showed that the shape of exosomes was round instead of cup. These techniques may combine structural analysis of proteins in exosomes using immunostaining, cryo-tomography, and sub-tomograms.

\section{CONFLICT OF INTEREST}

No potential conflict of interest relevant to this article was reported.

\section{ACKNOWLEDGMENTS}

EM studies were supported by Global Frontier Research Grant, NRF-2015M3A6A4065729, and supported by the Bio \& Medical Technology Development Program of the National Research Foundation (NRF) \& funded by the Korean government (MSIP\&MOHW) (No. 2016M3A9B6904244). This study was supported by the collaborative study program of National Institute for Physiological Sciences. We thank Jisoo Kim at Gyeongbuk Science \& Technology Promotion Center, Gumi Electronics \& Information Technology Research Institute for helping in obtaining serial images using FIBSEM. In addition, we also thank Dr. Oh Kyung Kwon of Nano Bioimaging Center at Seoul National University for cryoTEM images of exosomes. 


\section{REFERENCES}

Choi H, Jung M K, and Mun J Y (2017) Current status of automatic serial sections for 3D electron microscopy. Appl. Microsc. 47, 3-7.

Enderle D, Spiel A, Coticchia C M, Berghoff E, Mueller R, Schlumpberger M, Sprenger-Haussels M, Shaffer J M, Lader E, Skog J, and Noerholm M (2015) Characterization of RNA from exosomes and other extracellular vesicles isolated by a novel spin column-based method. PLoS One 10, e0136133.

Gyorgy B, Szabo T G, Pasztoi M, Pal Z, Misjak P, Aradi B, Laszlo V, Pallinger E, Pap E, Kittel A, Nagy G, Falus A, and Buzás E I (2011) Membrane vesicles, current state-of-the-art: emerging role of extracellular vesicles. Cell. Mol. Life Sci. 68, 2667-2688.

Jimenez N, Humbel B M, van Donselaar E, Verkleij A J, and Burger K N (2006) Aclar discs: a versatile substrate for routine high-pressure freezing of mammalian cell monolayers. J. Microsc. 221, 216-223.

Kim S B, Kim H R, Park M C, Cho S, Goughnour P C, Han D, Yoon I, Kim Y, Kang T, Song E, Kim P, Choi H, Mun J Y, Song C, Lee S, Jung H S, and Kim S (2017) Caspase-8 controls the secretion of inflammatory lysyltRNA synthetase in exosomes from cancer cells. J. Cell Biol. 216, 2201-2216.

Liu X and Wang H W (2011) Single particle electron microscopy reconstruction of the exosome complex using the random conical tilt method. J. Vis. Exp. doi: 10.3791/2574.

Montaner-Tarbes S, Borras F E, Montoya M, Fraile L, and Del Portillo H A (2016) Serum-derived exosomes from non-viremic animals previously exposed to the porcine respiratory and reproductive virus contain antigenic viral proteins. Vet. Res. 47, 59.
Raposo G and Stoorvogel W (2013) Extracellular vesicles: exosomes, microvesicles, and friends. J. Cell Biol. 200, 373-383.

Saari H, Lazaro-Ibanez E, Viitala T, Vuorimaa-Laukkanen E, Siljander P, and Yliperttula M (2015) Microvesicle- and exosome-mediated drug delivery enhances the cytotoxicity of Paclitaxel in autologous prostate cancer cells. J. Control. Release 220, 727-737.

Sharma S, Rasool H I, Palanisamy V, Mathisen C, Schmidt M, Wong D T, and Gimzewski J K (2010) Structural-mechanical characterization of nanoparticle exosomes in human saliva, using correlative AFM, FESEM, and force spectroscopy. ACS Nano 4, 1921-1926.

Thery C (2011) Exosomes: secreted vesicles and intercellular communications. F1000 Biol. Rep. 3, 15.

Thery C, Ostrowski M, and Segura E (2009) Membrane vesicles as conveyors of immune responses. Nat. Rev. Immunol. 9, 581-593.

Titze B and Genoud C (2016) Volume scanning electron microscopy for imaging biological ultrastructure. Biol. Cell 108, 307-323.

Villarroya-Beltri C, Baixauli F, Gutierrez-Vazquez C, Sanchez-Madrid F, and Mittelbrunn M (2014) Sorting it out: regulation of exosome loading. Semin. Cancer Biol. 28, 3-13.

Vlassov A V, Magdaleno S, Setterquist R, and Conrad R (2012) Exosomes: current knowledge of their composition, biological functions, and diagnostic and therapeutic potentials. Biochim. Biophys. Acta 1820, 940-948.

Zaborowski M P, Balaj L, Breakefield X O, and Lai C P (2015) Extracellular vesicles: composition, biological relevance, and methods of study. Bioscience 65, 783-797. 